\title{
Détermination rapide chez le chien et le lapin de l'urée et du glucose sanguins et urinaires
}

\author{
par J. P. PETIT
}

\begin{abstract}
RÉSUMÉ
L'expérimentation biologique utilise de plus en plus d'animaux, mais leurs normes biologiques restent souvent mal déterminées, empêchant une interprétation correcte des résultats.

C'est pourquoi, une méthode semi-quanfitative rapide de déterminatıon de l'urée et du glucose au moyen de bandelettes est proposée et testée quant à lo valeur qu'on peut lui attribuer. Dans le cas de chiens et de lapins, Il s'avère que la précision obtenue est suffisante pour permettre un irı rapide des animaux aptes à l'expérimentation. Les résultałs d'une comparaison de certains taux de glucose obtenus par un dosage enzymatique classique indiquent clairement qu'on peut substituer la méthode des bandelettes au dosage habituel, les moyennes obtenues par les deux méthodes ne diffèrent que de 1,5 à 1,66 p. 100.
\end{abstract}

\section{I. - INTRODUCTION}

A l'époque actuelle où les méthodes expérimentales de laboratoire font de plus en plus appel à l'utilisation d'animaux divers et à des techniques plus poussées et plus précises, il importe de connaître au plus près les caractéristiques essentielles des liquides organiques des sujets d'expérience par rapport à celles des sujets sains.

Ces données paraissent jusqu'ici avoir été ignorées ou délalssées, et cette ignorance peut expliquer certains résultats aberrants que rien ne semblait devoir justifier.

C'est pourquoi à l'occasion d'études sérologiques et biochimiques comparées, nous avons été amenés à nous préoccuper avant l'utilisation d'animaux d'expériences, de leur état bıologique de façon soit à éliminer ceux d'entre eux s'écartant par trop des normes dites «classiques». soit à en tenir compte dans l'interprétation des

(*) AZOSTIX et DEXTROSTIX, Laboratoires AMES : 80 , avenue Vicjor-Hugo, Paris $16 \mathrm{e}$. résultats, ce dernier procédé étant le plus délicat à mettre en œuvre.

Ces études ont surtout porté, chez le chien et le lapin, sur les possibilıtés pour eux d'avoir dans le sang ou l'urine un excès ou un défaut d'urée ou de glucose (*). Sans prétendre remplacer les méthodes classiques d'analyses, pratiquées habituellement pour ces dosages dans les laboratoires, méthodes parfaitement au point chez l'homme, beaucoup moins chez nos animaux domestiques ou expérimentaux, celle qui est présentée ici a l'avantage de pouvoir être pratıquée par l'expérımentateur lui-même à tout moment qu'il jugera opportun et sur une faible prise d'essai.

La simplıcité du procédé n'exclut pas une certaine rigueur dans les résultats ef c'est sur ce dernier point qu'il a paru particulièrement utıle d'insister en comparant quelques méthodes classiques d'analyses au procédé des bandelettes.

Il s'agit en effet d'utıliser des bandelettes toutes prêtes, imprégnées du réactif approprié pour effectuer des dosages semi-quantıtatıfs, le résultat recherché se situant dans une classe de valeurs 
sélectionnées parmi d'autres grâce d̀ une échelle colorimétrique.

Le principe est celvi, bien connu, des papiers indicateurs de $\mathrm{pH}$, modernisé dans sa conception et dans sa réalisation, puisque le support est en matière plastıque ef que certaines bandelettes permettent d'effectuer jusqu'à 5 déterminations $\left({ }^{*}\right)$.

\section{II. - Małériel utilisé}

Les estimations de l'urée et du glucose sanguins ont été respectivement faites avec les bandelettes Azostix et Dextrostix. La détermination précise de la glycémı a été obtenve en utılısant une méthode de dosage enzymatıque du glucose suivie d'une lecture spectrophotométrique sur PMQ II Zeiss.

Le temps a été mesuré au $1 / 5^{\mathrm{e}}$ de seconde avec un chronomètre de laboratoire, les diverses manipulations ont été réalisées de 22 à $28^{\circ} \mathrm{C}$, marge de température des locaux.

Les animaux sont des chiens de race commune et des lapins de races Buscat et Bélier ef leur croisement.

\section{III. - Méthodes et protocoles}

Le sang veineux est recueilli en tube sur héparine ef l'utilisation des bandelettes est faite dans les trente minutes suivantes.

Le. matérıel à utiliser est le sang complet et homogène dont il faut rapidement recouvrir entièrement la portion réactive de la bandelette avec une pipette. On attend alors exactement 60 secondes, puis on lave la bandelette avec le jet d'une pissette à eau distillée, dirigé juste audessus de la portion réactive. Dès ce moment, il faut faire très vite si on veut que les résultats soient précis et surtout reproductibles. II faut, immédiatement après le rinçage, éliminer l'excès d'eau et lire la coloration par rapport à l'échelle colorimétrique du flacon qui présente 4 plages de $0,20-0,45-0,85$ et $1,30 \mathrm{~g} / \mathrm{l}$ d'urée ou 5 plages de $0,45-0,90-1,30-1,75$ et 2,50 et plus $g / /$ de glucose.

On peut apprécier des plages intermédiaires

(*) LABSTIX, Laboratoires AMES, Recherche dans I'urıne des protéines, du glucose, des corps cétoniques et du sang, détermination du $\mathrm{pH}$. très rapidement, dès qu'on a pratiqué quelques dizaines de lectures.

\section{IV. - Résultafs ef discussion}

\section{Urémie chez le chien :}

Le tablequ I présente les résultats globaux obtenus sur les 14 animaux qui ont fait l'objet de ces recherches. Les dosages ont été pratiqués à différents intervalles sur les mêmes animaux pour suivre l'évolution de l'urémı au cours du temps chez un même animal.

TABLEAU I

Récapitulation du đosage de l'urée sanguine chez le chien

\begin{tabular}{|c|c|}
\hline $\begin{array}{c}\text { Effectif des classes } \\
\mathrm{n}_{\mathrm{i}}\end{array}$ & $\begin{array}{c}\text { Classes de } \mathrm{l}^{\prime} \text { 'urée } \\
\mathrm{eng} / \mathrm{ml} \\
\mathrm{x}_{\mathrm{i}}\end{array}$ \\
\hline 57,00 & 0,20 \\
6,00 & 0,32 \\
11,00 & 0,45 \\
1,00 & 0,60 \\
12,00 & 0,85 \\
\hline $\mathrm{N}=87,00$ & $\overline{\mathrm{x}}=0,33$ \\
\hline
\end{tabular}

Ce tableau regroupe 87 analyses et on peut observer la répartition bimodale de l'urée sanguine. Les deux maximums se situent aux alentours de $0,20 \mathrm{mg} / \mathrm{ml}$ et $0,85 \mathrm{mg} / \mathrm{ml}$, ce dernier mode étant le moins important, tandis que la moyenne générale vaut $0,33 \mathrm{mg} / \mathrm{ml}$, ce qui est normal.

L'écart type assez élevé rend compte des doses variées qu'on rencontre d'ordinaire chez le chien; avec les bandelettes nous trouvons comme valeurs extrêmes 0,20 et $0,85 \mathrm{mg} / \mathrm{ml}$, le calcul donne $0,33( \pm 0,44) \mathrm{mg} / \mathrm{ml}$ de sang.

Classiquement l'urée dosée par la méthode d̀ l'hypobromite voit son taux varier en moyenne de $0,20 \mathrm{mg} / \mathrm{ml}$ à $0,65 \mathrm{mg} / \mathrm{ml}$.

Chez un même animal on peuł noter une évoIution relativement régulière du taux de l'urée sanguine qui en général augmente avec le temps et les infestations parasitaires.

Le tableau II regroupe les analyses par animal avec les calculs des taux moyens d'urée et de glucose pour chacun d'entre eux.

Les valeurs trouvées sont proches des valeurs classiques avec une tendance à obtenir des 
TABIEAU II

Récapitulation des dosages effectués sur chaque chien

\begin{tabular}{|c|c|c|c|c|c|c|c|}
\hline \multirow{2}{*}{ Nom du chien } & \multirow{2}{*}{ Age } & \multicolumn{3}{|c|}{ UREE } & \multicolumn{3}{|c|}{ GLUCOSE } \\
\hline & & $\begin{array}{c}\text { Nombre } \\
\text { d'analyses }\end{array}$ & $\begin{array}{c}\text { Moyenne urée } \\
\text { en mg } / \mathrm{ml}\end{array}$ & Ecart type & $\begin{array}{c}\text { Nombre } \\
\text { d'analyses }\end{array}$ & $\begin{array}{c}\text { Moyenne glucose } \\
\text { en mg } / \mathrm{ml}\end{array}$ & Ecart type \\
\hline$J I X$ & 3 mois & 3 & 0,37 & 0,14 & 3 & 0,90 & 0,00 \\
\hline$J x$ & 3 mois & 3 & 0.32 & 0,12 & 3 & 3,80 & 4,50 \\
\hline $\mathrm{J} X I$ & 3 mois & 2 & 0,32 & 0,18 & 2 & 1,10 & 0,28 \\
\hline G V & 5 mois & 3 & 0,24 & 0,07 & 3 & 0,73 & 0,14 \\
\hline B VI & 5 mois & 5 & 0,20 & 0,00 & 5 & 0,96 & 0,34 \\
\hline B VII & 5 mois & 5 & 0,23 & 0,06 & 5 & 0,84 & 0,26 \\
\hline B VIII & 5 mois & 2 & 0,20 & 0,00 & 2 & 0,52 & 0,18 \\
\hline Blanchette & 5 mois & 8 & 0,25 & 0,09 & B & 0,79 & 0,18 \\
\hline G IV & 9 mois & 10 & 0,21 & 0,04 & 10 & 0,88 & 0,24 \\
\hline Pervenche & 3 ans 5 mois & 17 & 0,20 & 0,00 & 17 & 0,87 & 0,08 \\
\hline Zauzou & 4 ans 1 mois & 2 & 0,20 & 0,00 & 2 & 0,87 & 0,60 \\
\hline Papillon & 5 ans & 6 & 0,35 & 0,12 & 6 & 0,61 & 0,19 \\
\hline Fox & 5 ans & 9 & 0,23 & 0,08 & 9 & 0,65 & 0,18 \\
\hline Rita & 10 ans & 16 & 0,76 & 0,16 & 16 & 0,87 & 0,36 \\
\hline
\end{tabular}

valeurs un peu fortes en présence d'une quantité importante d'urée. En tout cas l'indication semiquantitative, donnée par les bandelettes, semble acceptable.

\section{$2^{\circ}$ Glucose sanguin chez le chien :}

TABLEAU III

Récapitulation du dosage du glucose sanguin chez le chien

\begin{tabular}{|c|c|}
\hline $\begin{array}{c}\text { Effectif des classes } \\
\mathrm{n}_{i}\end{array}$ & $\begin{array}{c}\text { Classes du glucose } \\
\text { en mg/m1 de sang } \\
\mathrm{x}_{\mathrm{i}}\end{array}$ \\
\hline 12,00 & 0,40 \\
1,00 & 0,48 \\
19,00 & 0,65 \\
2,00 & 0,72 \\
37,00 & 0,90 \\
6,00 & 1,10 \\
10,00 & 1,30 \\
\hline $\mathrm{N}=87,00$ & $\overline{\mathbf{x}}=0,69$ \\
\hline
\end{tabular}

Le tableau III regroupe les résultats. Le taux moyen ainsi obtenu : $0,69 \mathrm{mg} / \mathrm{ml}( \pm 0,62)$ est inférieur aux taux moyens trouvés par les méthodes non enzymatiques dans le sang total soit 0,82 à $1,01 \mathrm{mg} / \mathrm{ml}$ de sang. Les valeurs extrêmes trouvées sont éloıgnées, elles vont de 0,4 à $1,3 \mathrm{mg} / \mathrm{ml}$ ).
Il était donc intéressant de comparer ces valeurs avec celles obtenues par la méthode enzymatique et spectrophotométrique dans le tableau VI. Les moyennes obtenues diffèrent

\section{TABLEAU IV}

Conparaison des taux de glucose trouvés chez le lapin par la mêthode des bandelettes (mêthode 1) et par dosage enzymatique du glucose suivi d'une mesure spectrophotométrique (mêthode 2)

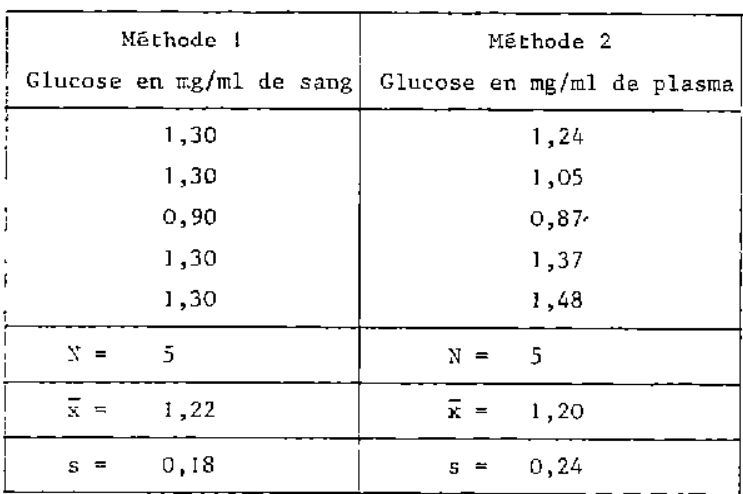

peu (1,5 p. 100), mais les valeurs extrêmes obtenues sont plus différentes dans le cas des bandelettes comme l'indique l'écart type plus élevé avec cette méthode 1 . Cecl n'est guère 


\section{TABLEAU $\mathrm{V}$}

Dosages de 1'uremie et de 1a glycëmie chez le lapin

\begin{tabular}{|c|c|c|c|c|}
\hline Race & $\mathbb{N}^{\circ}$ & Date de prëlèvement & Urëe en $\mathrm{mg} / \mathrm{ml}$ de sang & Glucose en $\mathrm{mg} / \mathrm{ml}$ de sang \\
\hline Bouscat & $\begin{array}{r}3 \\
6 \\
13 \\
18 \\
25\end{array}$ & $\begin{array}{r}2.4 .69 \\
22.4 .69 \\
2.4 .69 \\
26.3 .69 \\
22.4 .69\end{array}$ & \begin{tabular}{|c}
0,20 \\
Entre 0,20 et $0,45(0,33)$ \\
0,20 \\
Entre 0,20 et $0,45(0,33)$ \\
0,45
\end{tabular} & $\begin{array}{l}1,30 \\
1,30 \\
1,30 \\
0,90 \\
1,30\end{array}$ \\
\hline Bêlier & $\begin{array}{r}1 \\
14 \\
32\end{array}$ & $\begin{array}{l}26.3 .69 \\
16.4 .69 \\
26.3 .69\end{array}$ & $\begin{array}{c}0,20 \\
\text { Entre } 0,20 \text { et } 0,45(0,33) \\
0,20\end{array}$ & $\begin{array}{l}0,90 \\
1,30 \\
1,30\end{array}$ \\
\hline \multirow[t]{3}{*}{$\begin{array}{l}\text { Métis Bouscat } x \\
\text { Bêlier }\end{array}$} & $\begin{array}{l}15 \\
29\end{array}$ & $\begin{array}{l}22.4 .69 \\
22.4 .69\end{array}$ & $\begin{array}{l}0,90 \\
0,20\end{array}$ & $\begin{array}{l}0,45 \\
1,30\end{array}$ \\
\hline & & & $\begin{array}{l}\mathrm{N}=10,0 \\
\overline{\mathrm{x}}=\quad 0,33\end{array}$ & $\frac{10,0}{1,13}$ \\
\hline & & & 0,22 & 0,29 \\
\hline
\end{tabular}

TABLEAU VI

Comparaison du glucose sanguin dosê chez le chien par une mêthode enzymatique classique (méthode 2) et par les bandelettes imprégnées de réactif (méthode 1)

\begin{tabular}{|c|c|c|c|}
\hline \multirow{2}{*}{ Nom du chien } & \multirow{2}{*}{$\begin{array}{c}\text { Date quand il y a } \\
\text { plusieurs prêlèvements }\end{array}$} & Méthode 1 & Mêthode 2 \\
\hline & & $\begin{array}{l}\text { Glucose sanguin } \\
\text { en } \mathrm{mg} / \mathrm{ml}\end{array}$ & $\begin{array}{c}\text { Glucose plasmatique } \\
\text { en mg/m1 }\end{array}$ \\
\hline G IV & 23.1 .69 & 0,77 & 1,06 \\
\hline Pervenche & 23.1 .69 & 0,90 & 1,20 \\
\hline Rita & 23.1 .69 & 1,30 & 1,20 \\
\hline Fox & 23.1 .69 & 0,65 & 0,87 \\
\hline Pervenche & 30.1 .69 & 0,60 & 0,90 \\
\hline Rita & 30.1 .69 & 0,90 & 0,90 \\
\hline Blanchette & 30.1 .69 & 0.77 & 0,60 \\
\hline J IX & - & 0,90 & 0,90 \\
\hline $\mathrm{J} \mathrm{X}$ & - & 1,30 & 1,35 \\
\hline $\mathrm{J} X I$ & - & 1,30 & 1,12 \\
\hline Zouzou & - & 1,30 & 0,90 \\
\hline $\mathrm{N}$ & & 12 & 12 \\
\hline Taux moyen $\bar{x}$ en mg/m1 & & 0,96 & 0,97 \\
\hline Ecart type $s$ & & 0,27 & 0,22 \\
\hline
\end{tabular}


étonnant étant donné le caractère semi-quantitatif de la méthode.

La valeur indicatrice des bandelettes reste en moyenne utilisable, il fout cependant sıgnaler que dans certains cas on trouve des valeurs plus élevées et dans d'autres moins élevées que par un dosage enzymatique classique suivi d'une lecture spectrophotométrique.

\section{3o Urémie et glucose sanguin chez le lapin :}

Quoique avec 10 animaux seulement, l'expérience est statistiquement valable moyennant certaines précoutions relatives aux faibles échantillons.

Les résultats généraux sont réunis dans le tableau $V$, ils sont proches des normes généralement admises soit pour l'urée sanguine 0,36 à $0,72 \mathrm{mg} / \mathrm{ml}$ et pour le glucose par l'hypobromite 1,13 d̀ $1,15 \mathrm{mg} / \mathrm{ml}$, ici on trouve en moyenne $0,33 \mathrm{mg} / \mathrm{ml}( \pm 0,42)$ pour l'urée et $1,13 \mathrm{mg} / \mathrm{ml}$ $( \pm 0,58)$ pour le glucose.

Les comparaisons entre les dosages du glucose trouvé par la méthode enzymatique et par la méthode des bandelettes sont faites dans le tableau IV.

La plus grande dispersion des résultats dans le cas du dosage par la méthode 2 s'explıque: compte tenu de l'effectif, la précision est meilleure et les chiffres trouvés sont assez variés et non ordonnés en classes comme dans la méthode 1 .

Les moyennes trouvées sont cependant très voisines (égales à 1,66 p. 100 d'écart près).

\section{V. - Conclusions}

La comparasson des valeurs trouvées en utilisant les bandelettes et des valeurs admises en moyenne chez les chiens et les lapins indique claırement les possibilités de cette méthode, qui sont essentiellement d'assurer une orientation immédiate en indiquant un ordre de grandeur. Cette opinion est vérıfiée pour le glucose dans le cas d'une comparaison avec une méthade de dosage précise.

Les diverses pondérations qu'une expérimenfation plus poussée permettrait de conseiller ne sont pas souhaitables étant donné le caractère essentiel de cette méthode, qui est de donner une idée suffisamment précise pour permettre d'agir en conséquence immédiatement et non en moyenne avec 24 heures de délai, dans I'hypothèse d'une intervention thérapeutique. Pour ce qui est de la recherche biologique, on voit aisément qu'on peut ainsi trier les animaux aptes à servir dans une expérimentation biologique sans alourdir le protocole outre mesure étant donné la simplicité et surtout la rapidité de cette intervention supplémentaire.

D'autres constantes biologiques seront bientôt étudiées pour élargir le champ d'application de cette méthode.

Instifut d'Élevoge et de Médecine Vétérinaire des Poys tropicoux. Laboratoire de biochimie.

\section{SUMMARY}

Rapid determination of urinary and sanguine glucose and urea in dog and rabbif

Biological experiments need more and more animals whose biological standards often are badly determined, preventing a correct interpretation of results.

Therefore, a rapid semi-quantitative method of glucose and urea determination by means of reagent strips, is proposed and its possible value is tested.

With regard to dogs and rabbits, the precision obtained is proving sufficient for allowing the animals suitable fo the trial. The results of a comparison of some glucose rates obtained by a standard enzymatic fitration clearly show that the indicator paper method can replace the usual titration. The averages obtained by both methods differ only from 1,5 to 1,66 p. 100 .

\section{RESUMEN}

Determinación rápida de la urea y de la glucosa sanguîneas y urınarias en el perro y el conejo

La experimentación bıológica ufıliza cada vez mós animales, pero cuya normas bıológicas a menudo quedan mal determinadas, impediendo una interpretación exacta de los resultados. 
Por eso es por lo que se propone y se proba, en lo concerniente su valor, un método semi-cuantitativo rápido de determinación de la urea y de la glucosa mediante banda reactiva.

En lo concerniente los perros y los conejos, se comproba la precisión obtenida suficiente para permitir una selección rápida de los animales aptos para la experimentación. Los resultados de una comparación de ciertas tasas de glucosa obtenidas por un dosaje enzimatico habitual claramente indican que el método de la banda reactiva puede reemplazar el dosaje habitual; los terminos medios oblenidos por los dos métodos sólo varian de 1,5 à 1,66 p. 100. 